\title{
Dispersal limitation structures fungal community assembly in a long-term glacial chronosequence
}

\author{
Lauren C. Cline ${ }^{1 *}$ and Donald R. Zak ${ }^{1,2}$ \\ ${ }^{1}$ School of Natural Resources \& Environment and \\ ${ }^{2}$ Department of Ecology \& Evolution, University of \\ Michigan, 440 Church St., Ann Arbor, MI 48109, USA.
}

\section{Summary}

Microbial communities in soil mediate biogeochemical processes; however, understanding forces shaping their composition and function remains a gap in our ecological knowledge. We investigated phylogenetic turnover and functional gene composition of saprotrophic fungi along a 4000-year glacial chronosequence. A direct relationship between $\beta$-diversity and geographic distance, a proxy for time since deglaciation, indicated that dispersal limitation shapes saprotrophic fungal communities. Further, we infer that dispersal limitation may also influence fungal functional properties as metabolic potential and functional richness increased with site age. Despite attempts to minimize environmental variation, a direct relationship between $\beta$-diversity and biogeochemical differences across sites indicated that environmental filtering further shapes fungal community composition. However, environmental filtering was overshadowed by the effect of dispersal limitation when tested by multiple regression. Fungal $\beta$-diversity and composition of functional genes involved in plant litter decay were unrelated, suggesting that functional traits are not phylogenetically conserved across this chronosequence. Our study suggests that dispersal limitation operates in structuring present-day fungal community composition and functional potential. Further, we demonstrate the need to integrate functional and phylogenetic approaches to more accurately portray microbial communities and their functional capacities.

\section{Introduction}

As primary decomposers in terrestrial systems, understanding the ecological forces and timescales shaping

Received 2 July, 2013; revised 30 July, 2013; accepted 17 August, 2013. *For correspondence. E-mail clinela @umich.edu; Tel. (+1) 734709 9030; Fax (+1) 7349362195. saprotrophic microbial communities remains an important gap in our ecological knowledge. Identifying the rules by which soil microbial communities assemble has been hampered by their complexity, as well as prior conceptual and methodological limitations (Prosser et al., 2007). Due to their small size and rapid generation times, microbial communities were broadly believed to assemble swiftly in the absence of geographic or biological barriers to dispersal (Finlay, 2002). This perception resulted in the widespread hypothesis that with regard to microorganisms, 'everything is everywhere - the environment selects' (Baas Becking, 1934). Further, the remarkable diversity of soil microorganisms contributed to the belief that most are functionally equivalent.

In addition to environmental filtering, mounting evidence indicates that priority effects and dispersal limitation may shape microbial community assembly (Fukami et al., 2010; Martiny et al., 2011; Hanson et al., 2012). Over time, ecological drift differentiates communities that are dispersal-limited through stochastic local species extinctions (Vellend, 2010). In contrast, effective dispersal can lessen the strength of ecological drift as propagules from regional species pools enter a local community. Dispersal can also influence the sequence of colonist arrival, potentially shaping the trajectory of community assembly due to priority effects (Wilbur and Alford, 1985; Dickie et al., 2012). Environmental factors can act as 'filters', modifying community composition by constraining the occurrence of species or traits that make organisms poor competitors under specific conditions (Keddy, 1992). At a global scale, ecological drift may be a significant force because physical and ecological barriers limit gene flow among microbial communities (Papke and Ward, 2004). Physical and biotic factors appear to govern community composition at local landscapes (Edwards and Zak, 2010; Stegen et al., 2012). However, the interplay between historical processes and environmental filtering, particularly at intermediate spatial and temporal scales, remains unresolved (Martiny et al., 2011).

Despite recent developments, the relationship between microbial community composition and belowground processes is poorly understood (Van der Heijden et al., 2008). Due to physiological differences in the metabolism of organic substrates, rates of resource use and soil $\mathrm{C}$ storage may vary with shifts in microbial composition 
(Zhang et al., 2007; McGuire et al., 2010). Phylogenetic clustering of functional traits (Treseder et al., 2011; Martiny et al., 2012) provides a potential mechanism linking the composition of microbial communities to biogeochemical processes in soil. In contrast, others have demonstrated that ecosystem processes are robust to community differences due to an overlap in metabolic activity of organisms with varying evolutionary histories (Nielsen et al., 2011). The key to addressing this controversy is exploring the relationship between genetic identity and functional trait information (Hättenschwiler et al., 2011). With advances in molecular technologies, microbial ecologists have begun to characterize functional characteristics based on the presence of genes encoding enzymes that mediate key biogeochemical processes (He et al., 2007). Combining the study of microbial biogeography with functional trait analyses holds promise to increasing our mechanistic understanding of the environmental drivers of ecosystem-level processes, as well as the ecological forces structuring soil microbial communities.

Litter-decay fungi serve as a model system to investigate the functional biogeography of microorganisms involved in plant litter decomposition. Saprotrophic fungi mediate the decay of lignin, a major component of terrestrial plant litter (De Boer et al., 2005; Baldrian, 2006); moreover, they have the potential for long-distance dispersal and establishment as a result of spore production (Hallenberg and Kuffer, 2001). While recent studies have focused on the biogeography of plant-associated fungi (e.g. Kivlin et al., 2011; Tedersoo et al., 2012), few have considered community assembly dynamics of free-living saprotrophic fungi (Green et al., 2004; Feinstein and Blackwood, 2012). Despite evidence for long-distance wind dispersal of arctic ectomycorrhizal fungi (Bjorbækmo et al., 2010; Timling and Taylor, 2012), temperate fungal communities may show strong spatial structure (Edman et al., 2004; Peay et al., 2010). This observation suggests that historical processes, such as community assembly history and dispersal limitation, may have a lasting effect on saprotrophic fungal community composition.

To investigate the ecological mechanisms structuring fungal community composition and function, we quantified phylogenetic $\beta$-diversity and functional gene composition across a glacial chronosequence. Glacial retreat presents a unique opportunity to study the factors that structure composition and ecosystem properties through the formation of sites with varying ages yet similar attributes (e.g. Ohtonen et al., 1999). Retreat of the Wisconsin glacier ca. 14000 years ago occurred in a south to north direction across the Upper Great Lakes Region of North America. Distance between sites across this region, serving as a proxy for time since deglaciation, enabled us to investigate the impact of temporal gradients on the assembly of present-day fungal communities. Four northern hardwoods sites were selected along this chronosequence for similar edaphic and floristic characteristics to minimize environmental heterogeneity (Burton et al., 1991). Longterm monitoring of sites enabled us to quantify confounding variation along the temporal gradient, as well as investigate the impact of subtle environmental gradients on fungal community composition.

If dispersal limitation is at work at a regional scale, then a positive relationship should exist between fungal community dissimilarity and time since community assembly (i.e. distance between sites), independent of confounding environmental variation. Conversely, if environmental filtering is at work, then differences in fungal community composition should be related to environmental changes across sites. Furthermore, if community composition and function are linked, then communities that are phylogenetically similar will share a high degree of functional similarity. To test these hypotheses, we analysed fungal community composition by constructing clone libraries from forest floor DNA and profiled community functional potential using a functional gene microarray (He et al., 2007). Here, we provide evidence suggesting that dispersal limitation operates over the time frame of centuries to millennia to shape soil fungal communities.

\section{Results}

\section{Fungal community composition}

Following DNA extraction from forest floor samples, analysis of 1377 non-chimeric fungal sequences resulted in 721 unique sequences clustering within the subkingdom Dikarya, with an average sequence length of $\sim 900 \mathrm{bp}$. One hundred sixty-nine ascomycete and 129 basidiomycete operational taxonomic units (OTUs) were identified at $99 \%$ 28S sequence similarity. Ascomycete OTUs fell into the subphylum Pezizomycotina, whereas basidiomycete OTUs resided in subphylums Agaricomycotina and Pucciniomycotina. Good's coverage values from clone libraries ranged from $80 \%$ to $86 \%$, suggesting that the most abundant members of each community were sufficiently sampled, although some rare community members were not captured by our sampling effort.

Within each site, phylogenetic diversity (PD), net relatedness index (NRI) and nearest taxon index (NTI) were calculated (Faith, 1992) to characterize phylogenetic composition across our chronosequence. Phylogenetic diversity, the minimum branch length spanning a set of organisms on a phylogenetic tree, ranged from 1.89 to 2.20 among sites, and a one-way analysis of variance (ANOVA) indicated that PD did not differ among them (Table $1, P=0.61$ ). NRI ranged from 1.35 to 3.18 among sites in our chronosequence; it was significantly different 
Table 1. Metrics of phylogenetic community composition computed for each site.

\begin{tabular}{llll}
\hline Site & PD & NRI & NTI \\
\hline A & 1.89 & $3.18^{\mathrm{b}}$ & $3.42^{\mathrm{b}}$ \\
B & 2.20 & 1.35 & $2.12^{\mathrm{a}}$ \\
C & 1.79 & $2.28^{\mathrm{b}}$ & $3.17^{\mathrm{b}}$ \\
D & 2.16 & $3.11^{\mathrm{b}}$ & 0.66 \\
\hline
\end{tabular}

a. Indicates significant differences by comparison to a randomly generated phylogeny at $P<0.01$.

b. Denotes significant differences $P<0.001$.

PD represents phylogenetic diversity and NTI is nearest taxon index. PD was not significantly different across sites $(P=0.61)$. Significant $P$-values for NTI and NRI indicate fungal communities are more closely related than null communities.

from zero in Sites $A, C$ and $D(P<0.01)$, indicative of phylogenetic clustering. However, NRI in Site B was not significantly different from zero $(P=0.09)$. NTI ranged from 0.66 to 3.42 , and it was significantly greater than zero in Sites $A, B$ and $C(P<0.01)$, further indicating phylogenetic clustering within each community. The oldest site, Site D, did not exhibit phylogenetic clustering when considering its nearest neighbours because its NTI was indistinguishable from zero (Table 1, $P=0.260$ ). In combination, these observations indicated that fungal communities exhibit phylogenetic clustering along the chronosequence, although PD did not vary among sites.

Phylogenetic differences between communities composing our chronosequence were calculated by $\beta$-nearest taxon distance ( $\beta N T I)$ and Rao's phylogenetic distance $\left(D_{p}\right)$. The combination of metrics allowed $\beta$-diversity comparisons at two different phylogenetic resolutions (Stegen et al., 2012). For example, $\beta N T I$ quantified distance only between nearest relatives, whereas $D_{p}$ accounted for evolutionary relationships among taxa in both recent and deep evolutionary time. $\beta \mathrm{NTI}$ ranged from 0.71 to 3.65 for all pairwise comparisons of sites, and it was greatest between Sites $A$ and $D$ (the most distant sites), and smallest between Sites $C$ and $D$ (the closest sites; see Table S2). Despite variation in $\beta N T I$ across the chronosequence, $D_{p}$ narrowly ranged from 0.087 to 0.099 for all pairwise comparisons (Table S2), indicating that a small amount of unique branch length distinguished communities in our chronosequence. Taken together, fungal community along the chronosequence differed from one another in the occurrence of closely related species.

\section{Factors structuring phylogenetic $\beta$-diversity}

We modeled $\beta$-diversity as a function of pairwise differences in space (proxy for time) as well as environmental variation across the gradient (Anderson et al., 2011) in order to gain insight into the potential role of dispersal limitation and habitat filtering in structuring fungal composition (Fig. 2). $\beta N T I$ was significantly and positively related to log geographic distance $\left[P=0.003 ; r^{2}=0.91\right.$; Akaike's information criterion $(\mathrm{AIC})=7.75$ ] as well as biogeochemical distance $\left(P=0.04 ; \quad r^{2}=0.71 ; \quad A I C=14.86\right)$; geographic and biogeochemical distances were ztransformed in these analyses. In contrast, $\beta N T I$ was not related to soil climate $(P=0.97)$ or plant community composition $(P=0.71)$. Despite a weak correlation between geographic and biogeochemical distances $(P=0.09$; $r^{2}=0.48$; Table S2), the combination of spatial (i.e. site age) and biogeochemical factors predicted phylogenetic $\beta$-diversity with a higher goodness of fit $(P=0.01$; $r^{2}=0.95 ; \mathrm{AIC}=6.31$ ) than either factor alone in a linear regression model. Independent of factor order in the multiple regression model, the partial multiple regression coefficient for geographic distance did not differ from linear regression coefficient $(\beta=0.75 ; P=0.17)$, whereas the partial multiple regression coefficient for biogeochemical distance was slightly lower than that in the linear regression model $(\beta=0.30 ; P=0.06)$. Results demonstrate that confounding variation in biogeochemical properties does not independently drive the positive relationship between geographic distance, a proxy for site age, and fungal $\beta$-diversity. To gain a deeper understanding of the mechanism by which environmental variation structured fungal communities, biogeochemical variables were investigated independently using simple linear regression (Table 2). Following correction for false discovery rate (Benjamini and Hochberg, 1995), no single biogeochemical variable predicted $\beta$ NTI $(P=0.14-0.68)$.

\section{Functional potential of fungal communities}

Using a subset of gene probes on GEOCHIP 4.0 (i.e. genes synthesizing amylases, cellulases, chitinases, hemicellulases, ligninases, proteases), we quantified the physiological potential of fungal communities to metabolize plant and fungal detritus; these included functional

Table 2. Model ranking using Akaike's information criterion (AIC) where phylogenetic $\beta$-diversity ( $\beta N T I)$ is the response variable.

\begin{tabular}{lll}
\hline Model & AIC & $P$-value \\
\hline Annual ambient N deposition & 13.33 & 0.14 \\
Forest floor turnover & 15.74 & 0.18 \\
Litter N & 19.55 & 0.36 \\
Litter C & 19.85 & 0.36 \\
pH & 20.13 & 0.36 \\
Litter C:N & 20.65 & 0.39 \\
Litter mass & 21.94 & 0.68 \\
\hline
\end{tabular}

$P$-values were corrected for false discovery rate using the Benjamini and Hochberg correction. 


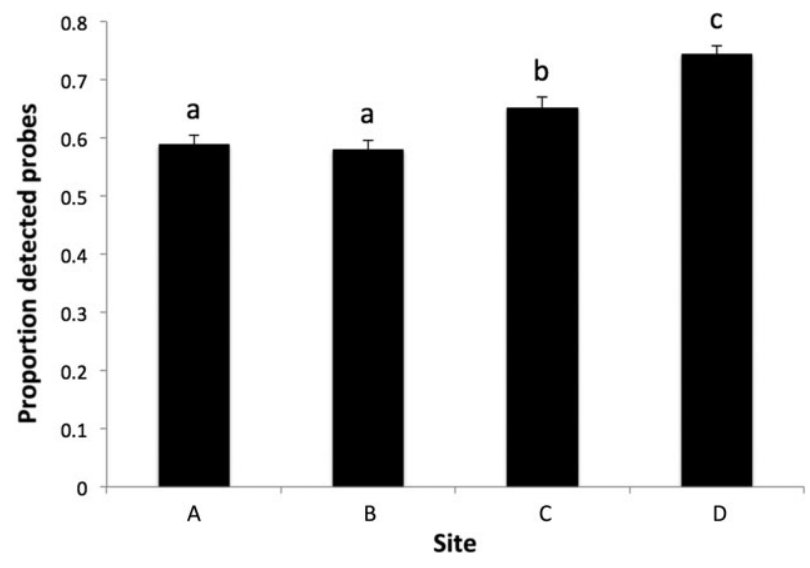

Fig. 1. Site functional richness calculated as average proportion of probes detected across all gene categories. Each functional gene has an equal weight within a gene category. Results of two-way ANOVA indicate significant main effects by site $\left(F_{3,48}=19.40\right.$; $P=0.002)$ and gene category $\left(F_{5,48}=4.40 ; P<0.001\right)$, but there was no significant interaction between them $\left(F_{14,48}=0.43 ; P=0.96\right)$. Error bars represent standard error. Lowercase letters represent statistical differences.

richness, metabolic potential and functional composition. Probe signal intensity (SI) was transformed to presenceabsence data to calculate functional richness. Two-way ANOVA revealed significant effects of site $\left(F_{3,48}=19.40\right.$; $P=0.002)$ and gene category $\left(F_{5,48}=4.40 ; P<0.001\right)$ on functional richness, but there was no significant interaction between them $\left(F_{14,48}=0.43 ; P=0.96\right)$. Post-hoc analyses revealed that functional richness, when averaged across gene category, was highest in the oldest Site D, followed by Site C and Site B. However, average functional richness of the youngest site, $\mathrm{A}$, was not significantly different from B (Fig. 1). As input for regression analysis, a distance matrix was generated using the Sørenson dissimilarity metric, in which the functional richness of each gene category was considered. Pairwise differences in functional richness ranged from $2.6 \%$ to $13.0 \%$.

Metabolic potential, calculated as corrected SI, significantly varied between sites (one-way ANOVA; $F_{3,8}=4.89$; $P=0.032$ ), and similarly increased with site age $\left(r^{2}=0.90 ; P=0.049\right.$; Fig. S1). Shapiro-Wilks test of normality indicated that data were normally distributed $(P=0.12)$, and equal variance was confirmed upon inspection of residual plots. A distance matrix of functional composition was generated by the Bray-Curtis dissimilarity metric. Pairwise site differences in functional composition ranged from $0.94 \%$ to $2.00 \%$.

\section{Factors structuring community functional potential}

We employed linear models to investigate the influence of spatial and environmental factors on the functional rich- ness of fungal genes involved in litter decay. Despite the increasing trend in functional richness across the chronosequence, pairwise site differences in functional richness did not significantly correlate to geographic distance $\left(r^{2}=0.03 ; P=0.74\right)$, biogeochemical distance $\left(r^{2}=0.11 ; P=0.51\right)$, soil climate $\left(r^{2}=0.59 ; P=0.07\right)$ or plant composition $\left(r^{2}=0.26 ; P=0.31\right)$. Because functional composition did not vary across the chronosequence, we did not relate spatial and environmental variation to differences in functional composition.

Despite patterned differences in $\beta$-diversity along the chronosequence, the relative abundance of functional genes involved in litter decay did not differ predictably among sites. In addition, functional composition was not related to $\beta N T I\left(r^{2}=0.13, P=0.49\right)$. Results from multivariate correlation test RELATE further indicated that no significant relationship occurred between $\beta$-diversity and pairwise differences in functional composition $(\rho=0.26$; $P=0.50)$.

\section{Discussion}

While microbial communities are thought to assemble rapidly in the absence of barriers to dispersal, we present evidence suggesting that dispersal limitation is a significant ecological force shaping saprotrophic fungal communities over the time frame of centuries to millennia. Evidence for dispersal limitation across our long-term chronosequence comes from the significant relationship we have documented between phylogenetic composition and time since deglaciation, wherein community dissimilarity increased along our spatial proxy for time since glacial retreat. Despite our attempts to minimize the influence of environmental filtering, fungal community dissimilarity also was influenced by biogeochemical variation among sites in our chronosequence. However, environmental filtering was overshadowed by the effect of dispersal limitation as revealed by our analyses. Dispersal limitation may also shape the functional potential of fungal communities, wherein functional richness and metabolic potential both increased with site age.

\section{Ecological factors structuring fungal communities}

While fungal spores have the potential to travel long distances (Favet et al., 2012), our findings indicate that temporal differences among sites differentiated fungal communities as a result of restricted dispersal. Phylogenetic differences in saprotrophic fungal communities increased with geographic distance, a surrogate for time since deglaciation, and accounted for more variation in community composition than biogeochemical distance alone (Fig. 2). One might argue that unmeasured environmental factors could underlie and covary with this 


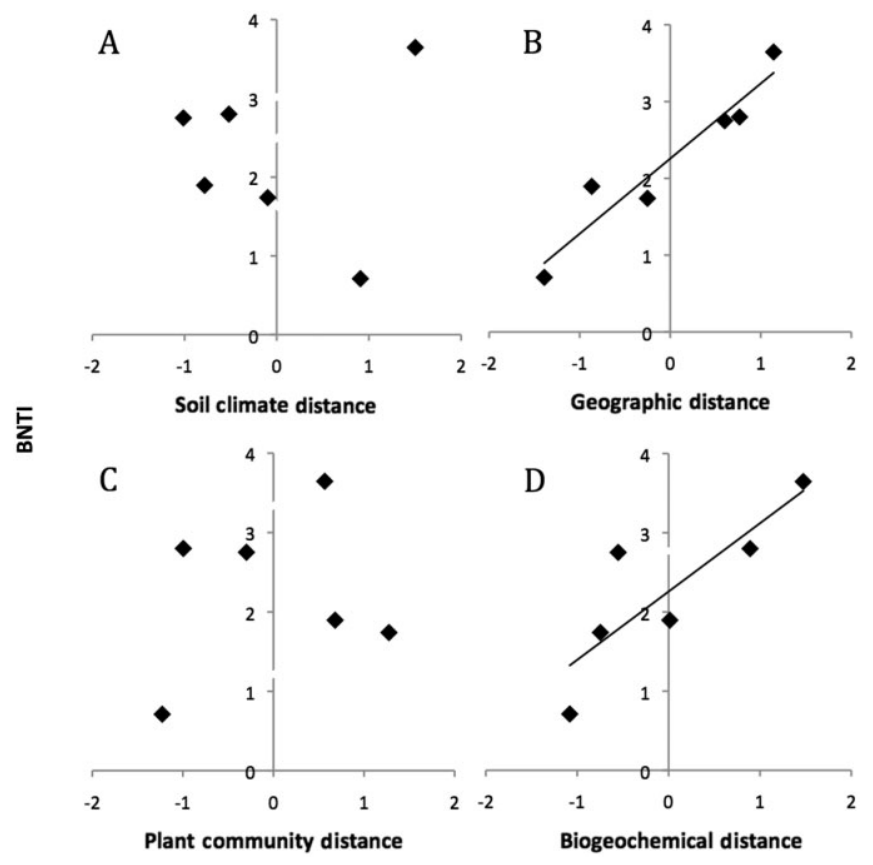

Fig. 2. Pairwise phylogenetic $\beta$-diversity ( $\beta N T I)$ plotted against soil climate distance $(A)$, geographic distance $(B)$, plant community distance $(C)$ and biogeochemical distance (D). All environmental distances were standardized to $z$-scores for meaningful comparison. Linear regression reveals a significant positive relationship between $\beta \mathrm{NTI}$ and geographic distance, as well as log biogeochemical distance at $P<0.05$. No significant relationship was found between $\beta \mathrm{NTI}$ and log soil climate distance $(P=0.96)$ or log plant community distance $(P=0.72)$.

relationship. However, our characterization of climate, plant community composition and biogeochemical properties minimizes this possibility. Widespread dispersal in arctic ectomycorrhizal fungi (Geml et al., 2012) indicates that the strength of geographic isolation may depend on habitat and fungal spore characteristics. The open, snow-covered arctic landscape potentially enhances spore transport by wind, whereas tree density in forests may limit long-distance fungal dispersal. Further, extreme environments, such as the arctic, may select fungi with long-distance dispersal capacities, such as small spore size and dark pigmentation (Timling and Taylor, 2012). Our findings challenge previous hypotheses regarding rapid community assembly and support the idea that restricted dispersal may have a persistent effect on microbial communities through time (Eisenlord et al., 2012).

Dispersal limitation and environmental filtering appear to work together to differentiate fungal communities in our chronosequence. Support for this assertion comes from the combination of time since deglaciation (i.e. distance) and biogeochemical characteristics providing a model with the highest goodness of fit for predicting phylogenetic $\beta$-diversity. Physiological adaptations for resource acquisition and defense mechanisms demonstrate that resource competition can influence fungal survival (Boddy, 2000; Lindahl and Olsson, 2004). Based on fundamental competition theory, organisms that are more efficient in resource capture outcompete and exclude organisms that are less efficient (Tilman, 1980). Subtle variation in biogeochemical characteristics across the chronosequence plausibly shaped traits for effective resource competition, assuming no variation or interaction with other factors. Results for environmental filtering are supported by evidence that fungal community composition is structured by biogeochemical factors, including soil $\mathrm{pH}$, ambient $\mathrm{N}$ deposition and litter biochemistry (Osono and Takeda, 2002; Zak et al., 2011; Hazard et al., 2013).

Historical processes may also govern the functional potential of fungal communities, demonstrated by increasing metabolic potential and functional gene richness across the chronosequence. However, we could not discern whether dispersal limitation or unmeasured environmental factors resulted in functional characteristics. Higher metabolic potential and functional richness in the older sites could be facilitated by adaptive radiation, as nucleotide substitutions created new functional gene variants, thereby increasing physiological diversity. Interestingly, a direct relationship between PD and site age was not observed when considering ribosomal genes as molecular markers. However, mutation rates of fungal ribosomal genes occur slowly over evolutionary time (Taylor and Berbee, 2006). It is possible that insufficient time has elapsed following deglaciation to allow radiation among colonizing organisms, unlike the evolutionary forces operating on functional genes as described above. Although differences in sampling completeness of the two molecular approaches may provide an alternative explanation, the relatively recent history of our study sites provides a more probable rationale to explain patterns of community richness in functional genes. As a result, older sites may have increased intraspecific variability with respect to the ability to enzymatically harvest energy from plant and fungal detritus.

Functional richness might have consequences for ecosystem processes by providing more opportunities for efficient resource use in a variable environment (e.g. niche complementary effect) or increasing the probability that the presence of a functional trait will dominate ecosystem functioning (e.g. selection effect; Loreau, 1998). Empirical support for this idea comes from a grassland ecosystem, in which rates of soil organic matter accumulation were positively related to functional gene richness (Zhang et al., 2007). Our reported increase in metabolic potential and functional richness of the fungal community to degrade plant litter provides further support for this assertion. 
Table 3. Site location, age, soil climate and biogeochemical soil characteristics.

\begin{tabular}{|c|c|c|c|c|}
\hline Site characteristics & A & B & C & $\mathrm{D}$ \\
\hline Location & $46^{\prime} 51^{\prime \prime} \mathrm{N} ; 88^{\prime} 52^{\prime \prime} \mathrm{W}$ & $45^{\prime} 32^{\prime \prime} \mathrm{N} ; 84^{\prime} 51^{\prime \prime} \mathrm{W}$ & $44^{\prime} 22^{\prime \prime} \mathrm{N} ; 85^{\prime} 49^{\prime \prime} \mathrm{W}$ & $43^{\prime} 40^{\prime \prime} \mathrm{N} ; 86^{\prime} 08^{\prime \prime} \mathrm{W}$ \\
\hline Glacial retreat (years before present) & 9500 & 11000 & 13000 & 13500 \\
\hline \multicolumn{5}{|l|}{ Soil climate } \\
\hline Average soil temperature $\left({ }^{\circ} \mathrm{C}\right)$ & 7.2 & 7.9 & 8.3 & 9.1 \\
\hline Average soil matric potential (MPa) & -0.14 & -0.15 & -0.14 & -0.18 \\
\hline \multicolumn{5}{|l|}{ Biogeochemical characteristics } \\
\hline Soil pH & 4.55 & 4.70 & 4.41 & 4.61 \\
\hline Forest floor $\mathrm{N}$ content $\left(\mathrm{g} \mathrm{N} \mathrm{m}^{-2}\right)$ & 14.3 & 32.1 & 25.9 & 40.6 \\
\hline Forest floor C:N & 63.7 & 57.1 & 52.9 & 43.4 \\
\hline Forest floor turnover $(\mathrm{y})$ & 2.21 & 4.91 & 5.16 & 6.48 \\
\hline Ambient $\mathrm{N}$ deposition $\left(\mathrm{kg} \mathrm{N} \mathrm{ha}^{-1} \mathrm{y}^{-1}\right)$ & 5.89 & 6.07 & 7.37 & 7.37 \\
\hline
\end{tabular}

See Supporting information (Table S1) for plant community composition data.

\section{Link between community composition and functional potential}

Contrary to our predictions, we did not observe a direct link between functional gene composition and fungal $\beta$-diversity. One potential explanation is that the collective suite of functional genes involved in litter decay was not phylogenetically conserved among saprotrophic fungi in the soils of our chronosequence. However, certain capabilities, including ligninolytic decay, are known to be constrained in saprotrophic fungi of the Dikarya subkingdom (Baldrian, 2006; Osono, 2007), indicating that functional traits can in fact be conserved for this group of organisms. Metabolically less complex components of litter decay may have evolved independently within saprotrophic fungi. Alternatively, constrained variation in plant communities may explain the lack of correlation between functional gene composition and fungal community composition. By minimizing variation in plant community composition and biogeochemical factors, our experimental design may also have constrained functional composition via the effect of plant litter biochemistry on saprotrophic metabolism. The aforementioned observations do not necessarily dispel the hypothesis that community composition has functional consequences. Instead, community assembly rules may vary based upon the level of community organization (Fukami et al., 2005). While our study investigated the links between composition and functional potential, enzyme synthesis via transcription and translation also contributes to biogeochemical processes. Closer investigation of gene regulation and rates of soil $\mathrm{C}$ cycling may be required to capture the complete linkage between community composition and function.

\section{Conclusion}

In summary, our study suggests that dispersal limitation is an ecological force shaping the composition and functional potential of litter-decay fungi in a long-term chronosequence. The persistent effect of dispersal limita- tion on fungal community assembly challenges previous conceptions of ubiquitous dispersal and rapid community assembly. Dispersal limitation in ectomycorrhizal fungi (Peay et al., 2010), as well as filamentous bacteria (Eisenlord et al., 2012), for example, adds additional support to the notion that this ecological mechanisms shape the composition and function of various groups of soil microorganisms. Improved knowledge of fungal dispersal capabilities as well as future manipulative studies are needed to directly test the influence of dispersal limitation on fungal community assembly. Nonetheless, our results highlight the importance of dispersal limitation as an ecological agent shaping community composition and functional traits of saprotrophic fungal communities in temperate forests.

\section{Experimental procedures}

\section{Study sites}

Forest floor samples were collected in October 2009 from four sugar maple (Acer saccharum Marsh.) dominated northern hardwood sites. Study sites span $400 \mathrm{~km}$ south to north along Lower and Upper Michigan, USA. Locations were selected from a population of 31 candidate sites based on floristic and edaphic similarity (Burton et al., 1991). Soils at these sites are well-drained sandy typic Haplothords of the Kalkaska series. For additional site information, see Tables 3 and S1. At each site, forest floor samples were collected from three $30 \times 30 \mathrm{~m}$ replicate plots. Within each plot, 10 random $10 \times 10 \mathrm{~cm}$ forest floor samples were collected and composited after manually removing the Oi horizon. In each plot, Oe and Oa horizons were homogenized by hand with sterilized scissors, and a $10 \mathrm{~g}$ subsample was taken for laboratory analysis. A total of 12 samples were flash-frozen in $\mathrm{N}_{2}$ liquid, and were immediately transported to the University of Michigan and stored at $-80^{\circ} \mathrm{C}$.

\section{DNA extraction, amplification and molecular cloning}

Genomic DNA was extracted from $2.5 \mathrm{~g}$ of forest floor material using PowerMax TM Soil Extraction Kits (Mo Bio Labo- 
ratories, Solana Beach, CA, USA). Fungal rDNA was selectively amplified using primers 58AF2 (Martin and Rygiewicz, 2005) and LR3 (Hopple and Vilgalys, 1994) targeting a fragment of the internal transcribed spacer region (ITS-2) and large ribosomal segment (28S gene). Duplicate polymerase chain reactions (PCR) for each composite forest floor sample contained $1 \mu \mathrm{l}$ of DNA, $0.4 \mu \mathrm{l}$ of $10 \mu \mathrm{M}$ forward primer 58AF2, $0.4 \mu \mathrm{l}$ of $10 \mu \mathrm{M}$ reverse primer LR3, $2.5 \mu \mathrm{l}$ dNTPs $(2 \mu \mathrm{M}), 2.5 \mu \mathrm{l} 10 \times$ PCR buffer (1.5 mM MgCl 2 ; Roche, Hamburg, Germany), $1 \mu$ I BSA, $0.5 \mu$ l high-fidelity Taq polymerase (Roche), and $16.7 \mu \mathrm{l}$ molecular grade water. Duplicate PCR reactions ensured great enough mass of the targeted region for downstream analysis. After an initial denaturation step of $1 \mathrm{~min}$ at $95^{\circ} \mathrm{C}$, cycles of denaturing at $95^{\circ} \mathrm{C}$ for $30 \mathrm{~s}$, annealing at $50^{\circ} \mathrm{C}$ for $30 \mathrm{~s}$ and elongation at $72^{\circ} \mathrm{C}$ for $60 \mathrm{~s}$, followed by a final extension step of $72^{\circ} \mathrm{C}$ for $15 \mathrm{~min}$, was carried out using Eppendorf PCR cyclers (Hamburg, Germany). Duplicate PCR products were combined and purified (UltraClean PCR Clean-up, Mo Bio), followed by cloning into pCR 2.1-TOPO using the TOPO TA Cloning kit (Invitrogen, Grand Island, NY, USA). Vectors were transformed into TOP10 competent cells. One hundred and ninety two clones were selected from each plot and grown overnight in 96 well plates at $37^{\circ} \mathrm{C}$ in Luria-Bertani broth containing $10 \%$ glycerol, $0.025 \mathrm{~g} \mathrm{l}^{-1}$ ampicillin, $0.0125 \mathrm{~g} \mathrm{l}^{-1}$ kanamycin. Libraries were submitted for Sanger sequencing at SeqWright (Houston, TX, USA).

\section{Phylogenetic analyses}

Sequence histograms were edited, and contiguous sequences were constructed using GenEIOUS 5.4.2 (Kearse et al., 2012) for the 12 clone libraries. Clone and reference sequences were aligned with MAFFT in GENEIOUS (Katoh et al., 2002), and manually edited for gaps and hypervariable regions. Closest reference sequences were identified using a BLAST search (Altschup et al., 1990) and incorporated into the alignment. Suspected chimeric sequences were manually identified due to extreme variation in length, non-continuous sequences or grouping with non-fungal reference sequences on preliminary neighbour-joining trees constructed in GENEIOUS. Similarity matrices were generated based on alignments of the homologous $28 \mathrm{~S}$ region, followed by grouping OTUs based on $99 \%$ sequence similarity using the average neighbour algorithm in MOTHUR (Schloss et al., 2009; Edwards and Zak, 2010). Sequences representing each OTU have been deposited in GenBank (KC588534$\mathrm{KC588833).} \mathrm{OTUs} \mathrm{were} \mathrm{aligned} \mathrm{with} \mathrm{basidiomycete} \mathrm{and}$ ascomycete reference sequences, and a Glomeromycete outgroup (see Table S4) using MAFFT in GenEIOUS. A maximum likelihood tree was constructed using PHYML in MEGA 5.0 with generalized time reversible selected as the best substitution model using JMODELTEST (Posada and Crandall, 1998; Tamura et al., 2011). This tree was used as input for community analyses based on branch length (see Fig. S3).

\section{Community composition and structure}

Phylogenetic composition within and between communities was quantified using PHYLOCOM version 4.2 (Webb et al.,
2008). Phylogenetic diversity (PD) is defined as the minimum branch length spanning any given set of species on a phylogenetic tree. The NTI quantified the standard deviation of phylogenetic distance between closest relatives in a community when compared with the phylogenetic distance of closest relatives in a community randomly assembled from the tips of the phylogeny (999 randomizations). The NRI is calculated as a tree-wide measure of phylogenetic clustering. $\mathrm{NTI}$ and NRI scores significantly greater than zero suggest that communities are more closely related than expected by chance, while negative scores suggest that communities are more distantly related than expected by chance (Webb et al., 2002).

Investigating phylogenetic distance between pairs of communities provided information about $\beta$-diversity across distance and environmental gradients. $D_{p}$ represents an abundance-weighted measure of the pairwise differences in branch length of OTUs in a community relative to another. A higher $D_{p}$ for an assemblage is indicative of higher PD relative to the total taxa pool, and therefore more phylogenetically distant pairs. $\beta \mathrm{NTI}$, the between-community analogue to NTI, is the deviation of the abundance-weighted phylogenetic distance among the closest relatives between two communities $\left(\beta M N T D_{\text {obs }}\right)$ as compared with the average phylogenetic distance when both communities are randomized across the phylogenetic tree (mean $\beta M N T D_{\text {null }}$ ). A null distribution was found by shuffling species labels across the phylogeny, the 'phylogeny shuffle' null model in PHYLOCOM, and recalculating BMNTD 999 times. Community turnover increases as $\beta$ NTI departs from the mean of this distribution, providing support for the role of abiotic or biotic forces in structuring $\beta$-diversity (Stegen et al., 2012). Because $D_{p}$ accounts for evolutionary relationships among taxa in both recent and evolutionary time, whereas $\beta \mathrm{NTI}$ quantifies distance only between nearest relatives, we believe that these approaches provided a more complete understanding of community differences.

\section{GEOCHIP 4.0 processing and analyses}

Analysis of the metabolic potential of the saprotrophic fungal community was conducted using the GEOCHIP 4.0 (He et al., 2007). One microgram of genomic DNA from each replicate sample was purified by the Genomic DNA Clean \& Concentrator kit (Zymo Research, Irvine, CA, USA) and labelled with fluorescent dye Cy-3 using random primers (Wu et al., 2006). The labelled gDNA was dried and rehydrated with $2.7 \mu \mathrm{l}$ of sample tracking control, followed by incubation at $50^{\circ} \mathrm{C}$ for 5 min. This DNA solution was then mixed with $7.3 \mu \mathrm{l}$ of hybridization buffer containing the universal standard DNA labelled with $\mathrm{Cy}-5$ dye; it was denatured at $95^{\circ} \mathrm{C}$ for 5 min and maintained at $42^{\circ} \mathrm{C}$ until loaded onto GEOCHIP arrays (NimbleGen, Madison, WI, USA). The hybridization was performed on a Hybridization Station (MAUI, Roche, CA, USA) at $42^{\circ} \mathrm{C}$ for $16 \mathrm{~h}$ with agitation. After washings, the arrays were scanned using an MS 200 Microarray Scanner (NimbleGen).

To investigate functional potential of the fungal community to degrade plant and fungal litter, we selected a subset of probes on GEOCHIP 4.0. Six gene categories were identified from the 'carbon cycling' genes based on the metabolism of specific substrates, including amylases, cellulases, 
chitinases, hemicellulases, lignininases and proteases; only fungal functional genes were analysed. Across all gene categories, 31 functional genes and a total of 1398 probes were used in analysis of functional potential (see Table S3). Functional richness, the number of targeted gene variants encoding the same function (i.e. lignin depolymerization), represented the number of targeted probes within a gene category registering a fluorescent signal greater than zero. Overall metabolic potential was calculated as the sum of corrected fluorescent SI of the selected 31 functional genes. Functional composition was calculated as the relative abundance of functional genes within each gene category. Varying proportions of functional genes within each gene category may suggest varying physiological abilities of the fungal community to metabolize plant and fungal detritus. Fluorescent SI and richness calculations were corrected by the number of probes per gene, and genes per gene category; therefore, individual genes and gene categories were not weighted by an uneven abundance of probes or functional genes on the microarray (Xie et al., 2011).

Differences in total metabolic potential and functional richness across the chronosequence were explored using ANOVA in the software package R 2.11 (http://www.rproject.org). Post-hoc analyses were conducted using Tukey's honestly significant difference (HSD) test. All assumptions of linearity were verified prior to analysis by Shapiro-Wilks tests and inspection of residual plots. To investigate the factors contributing to site differences in functional richness and composition of the six functional gene categories involved in litter decay, two distance matrices were created. Pairwise comparisons of functional richness were calculated by the Sørenson dissimilarity metric. The BrayCurtis dissimilarity metric was used to generate differences in functional composition across sites.

\section{Environmental analyses}

Environmental variables were assembled into three datasets: (i) soil climate, (ii) biogeochemical characteristics and (iii) plant community composition. Soil climate data consisted of average annual soil temperature and soil matric potential. Data were collected using data loggers at each plot, and reflect averages between 1988 and 2009. Soil climate was chosen because soil variables more closely reflect the environment saprotrophic fungi experience relative to ambient air temperature and precipitation. The biogeochemical dataset consisted of previously collected values of soil $\mathrm{pH}$, forest floor $\mathrm{N}$ content $\left(\mathrm{g} \mathrm{N} \mathrm{m}^{-2}\right.$ ), forest floor $\mathrm{C}: \mathrm{N}$, leaf litter forest floor turnover (years), and annual ambient $\mathrm{N}$ deposition ( $\mathrm{g} \mathrm{N} \mathrm{m}^{-2} \mathrm{y}^{-1}$ ) from 2005 to 2009 (Table 3). Plant community composition was calculated as the relative dominance based on basal area of overstory species at the site (Table S1). Soil climate and biogeochemical dissimilarity matrices were generated with Euclidean distances of log-transformed data. The plant community dissimilarity matrix was generated using the Bray-Curtis metric (Bray and Curtis, 1957).

Regression analyses were used to investigate the important environmental variables that contributed to differences in fungal phylogenetic $\beta$-diversity and functional richness (Anderson et al., 2011; Fine and Kembel, 2011; Stegen et al., 2012). Simple and multiple linear regression was used to quantify pairwise community differences attributable to geographic distance (a surrogate for time), plant community composition, as well as soil climate and biogeochemical characteristics across sites. Regression analysis was selected as most appropriate for our dataset, as the partial Mantel test underestimates the amount of variation in community composition using dissimilarity matrices (Legendre et al., 2005), and we lacked statistical power $(n=4)$ to conduct redundancy or canonical analysis that considered more than one variable at a time. Raw environmental distances were standardized to z-scores prior to regression, and AIC was used to compare linear models. All assumptions of linearity were verified prior to analysis. When there was a significant relationship between an environmental category (e.g. biogeochemical) and phylogenetic or functional distance, we evaluated the relative importance of individual environmental factors (e.g. soil $\mathrm{pH}$ ) in structuring community differences. Regression was also used to test the relationship between $\beta$-diversity and functional composition.

\section{Acknowledgements}

This research was made possible by support for the National Science Foundation's Long-Term Research in Environmental Biology programme, and the Department of Energy's Biological and Environmental Research Division. The authors would like to thank Sarah Eisenlord and Rima Upchurch for help in sampling and method development, and Tong Yuan from Jizhong Zhou's lab for her work in running the microarray.

\section{References}

Altschup, S.F., Gish, W., Miller, W., Myers, E.W., and Lipman, D.J. (1990) Basic local alignment search tool. J Mol Biol 215: 403-410.

Anderson, M.J., Crist, T.O., Chase, J.M., Vellend, M., Inouye, B.D., Freestone, A.L., et al. (2011) Navigating the multiple meanings of $\beta$ diversity: a roadmap for the practicing ecologist. Ecol Lett 14: 19-28.

Baas Becking, L.G.M. (1934) Geobiologie of Inleiding Tot De Milieukunde. The Hague, the Netherlands: W.P. Van Stockum \& Zoon (in Dutch).

Baldrian, P. (2006) Fungal laccases - occurrence and properties. FEMS Microbiol Rev 30: 215-242.

Benjamini, Y., and Hochberg, Y. (1995) Controlling the false discovery rate: a practical and powerful approach to multiple testing. J R Stat Soc Ser B 57: 289-300.

Bjorbækmo, M.F.M., Carlsen, T., Brysting, A., Vrålstad, T., Høiland, K., Ugland, K.I., et al. (2010) High diversity of root associated fungi in both alpine and arctic Dryas octopetala. BMC Plant Biol 10: 244.

Boddy, L. (2000) Interspecific combative interactions between wood-decaying basidiomycetes. FEMS Microbiol Ecol 31: 185-194.

Bray, J.R., and Curtis, J.T. (1957) An ordination of upland forest communities of southern Wisconsin. Ecol Monogr 27: 325-349.

Burton, A.J., Ramm, C.W., and Pregitzer, K.S. (1991) Use of multivariate methods in forest research site selection. Can J For Res 21: 1573-1580. 
De Boer, W., Folman, L.B., Summerbell, R.C., and Boddy, L. (2005) Living in a fungal world: impact of fungi on soil bacterial niche development. FEMS Microbiol Rev 29: 795-811.

Dickie, I.A., Fukami, T., Wilkie, J.P., Allen, R.B., and Buchanan, P.K. (2012) Do assembly history effects attenuate from species to ecosystem properties? A field test with wood-inhabiting fungi. Ecol Lett 15: 133-141.

Edman, M., Kruys, N., and Jonsson, B.G. (2004) Local dispersal sources strongly affect colonization patterns of wood-decaying fungi on spruce logs. Ecol Appl 14: 893901.

Edwards, I.P., and Zak, D.R. (2010) Phylogenetic similarity and structure of Agaricomycotina communities across a forested landscape. Mol Ecol 19: 1469-1482.

Eisenlord, S.D., Zak, D.R., and Upchurch, R.A. (2012) Dispersal limitation and the assembly of soil Actinobacteria communities in a long-term chronosequence. Ecol Evol 2: 538-549.

Faith, D.P. (1992) Conservation evaluation and phylogenetic diversity. Biol Conserv 61: 1-10.

Favet, J., Lapanje, A., Giongo, A., Kennedy, S., Aung, Y., Cattaneo, A., et al. (2012) Microbial hitchhikers on intercontinental dust: catching a lift in Chad. ISME J 7: 850867.

Feinstein, L.M., and Blackwood, C.B. (2012) Taxa-area relationship and neutral dynamics influence the diversity of fungal communities on senesced tree leaves. Environ Microbiol 14: 1488-1499.

Fine, P.V., and Kembel, S.W. (2011) Phylogenetic community structure and phylogenetic turnover across space and edaphic gradients in western Amazonian tree communities. Ecography 34: 552-565.

Finlay, B.J. (2002) Global dispersal of free-living microbial eukaryote species. Science 296: 1061-1063.

Fukami, T., Bezemer, T.M., Mortimer, S.R., and Van der Putten, W.H. (2005) Species divergence and trait convergence in experimental plant community assembly. Ecol Lett 8: 1283-1290.

Fukami, T., Dickie, I.A., Wilkie, J.P., Paulus, B.C., Park, D., Roberts, A., et al. (2010) Assembly history dictates ecosystem functioning: evidence from wood decomposer communities. Ecol Lett 13: 675-684.

Geml, J., Timling, I., Robinson, C.H., Lennon, N., Nusbaum, H.C., Brochmann, C., et al. (2012) An arctic community of symbiotic fungi assembled by long-distance dispersers: phylogenetic diversity of ectomycorrhizal basidiomycetes in Svalbard based on soil and sporocarp DNA. J Biogeogr 39: 74-88.

Green, J.L., Holmes, A.J., Westoby, M., Oliver, I., Briscoe, D., Dangerfield, M., et al. (2004) Spatial scaling of microbial eukaryote diversity. Nature 432: 747-750.

Hallenberg, N., and Kuffer, N. (2001) Long-distance spore dispersal in wood-inhabiting basidiomycetes. Nord $J$ Bot 21: 431-436.

Hanson, C.A., Fuhrman, J.A., Horner-Devine, M.C., and Martiny, J.B.H. (2012) Beyond biogeographic patterns: processes shaping the microbial landscape. Nat Rev Microbiol 10: 497-506.

Hättenschwiler, S., Fromin, N., and Barantal, S. (2011)
Functional diversity of terrestrial microbial decomposers and their substrates. C R Biol 334: 393-402.

Hazard, C., Gosling, P., van der Gast, C.J., Mitchell, D.T., Doohan, F.M., and Bending, G.D. (2013) The role of local environment and geographical distance in determining community composition of arbuscular mycorrhizal fungi at the landscape scale. ISME J 7: 498-508.

He, Z., Gentry, T.J., Schadt, C.W., Wu, L., Liebich, J., Chong, S.C., et al. (2007) GeoChip: a comprehensive microarray for investigating biogeochemical, ecological and environmental processes. Environ Sci 1: 67-77.

Hopple, J.S., and Vilgalys, R. (1994) Phylogenetic relationships among coprinoid taxa and allies based on data from restriction site mapping of nuclear rDNA. Mycologia 86: 96-107.

Katoh, K., Misawa, K., Kuma, K., and Miyata, T. (2002) MAFFT: a novel method for rapid multiple sequence alignment based on fast Fourier transform. Nucleic Acids Res 30: 3059-3066.

Kearse, M., Moir, R., Wilson, A., Stones-Havas, S., Cheung, M., Sturrock, S., et al. (2012) Geneious basic: an integrated and extendable desktop software platform for the organization and analysis of sequence data. Bioinformatics 28: $1647-1649$.

Keddy, P.A. (1992) Assembly and response rules: two goals for predictive community ecology. J Veg Sci 3: 157164.

Kivlin, S.N., Hawkes, C.V., and Treseder, K.K. (2011) Global diversity and distribution of arbuscular mycorrhizal fungi. Soil Biol Biochem 43: 2294-2303.

Legendre, P., Borcard, D., and Peres-Neto, P. (2005) Analyzing beta diversity: partitioning the spatial variation of community composition data. Ecol Monogr 75: 435450.

Lindahl, B.D., and Olsson, S. (2004) Fungal translocation creating and responding to environmental heterogeneity. Mycologist 18: 79-88.

Loreau, M. (1998) Biodiversity and ecosystem functioning: a mechanistic model. Proc Natl Acad Sci USA 95: 56325636.

McGuire, K.L., Bent, E., Borneman, J., Majumder, A., Allison, S.D., and Treseder, K.K. (2010) Functional diversity in resource use by fungi. Ecology 91: 2324-2332.

Martin, K.J., and Rygiewicz, P.T. (2005) Fungal-specific PCR primers developed for analysis of the ITS region of environmental DNA extracts. BMC Microbiol 5: 28.

Martiny, A.C., Treseder, K.K., and Pusch, G. (2012) Phylogenetic conservatism of functional traits in microorganisms. ISME J 7: 830-838.

Martiny, J.B.H., Eisen, J.A., Penn, K., Allison, S.D., and Horner-Devine, M.C. (2011) Drivers of bacterial $\beta$-diversity depend on spatial scale. Proc Natl Acad Sci USA 108: 7850-7854.

Nielsen, U.N., Ayres, E., Wall, D.H., and Bardgett, R.D. (2011) Soil biodiversity and carbon cycling: a review and synthesis of studies examining diversity-function relationships. Eur J Soil Sci 62: 105-116.

Ohtonen, R., Fritze, H., Pennanen, T., Jumpponen, A., and Trappe, J. (1999) Ecosystem properties and microbial community changes in primary succession on a glacier forefront. Oecologia 119: 239-246. 
Osono, T. (2007) Ecology of ligninolytic fungi associated with leaf litter decomposition. Ecol Res 22: 955-974.

Osono, T., and Takeda, H. (2002) Comparison of litter decomposing ability among diverse fungi in a cool temperate deciduous forest in Japan. Mycologia 94: 421-427.

Papke, R.T., and Ward, D.M. (2004) The importance of physical isolation to microbial diversification. FEMS Microbiol Ecol 48: 293-303.

Peay, K.G., Kennedy, P.G., Davies, S.J., Tan, S., and Bruns, T.D. (2010) Potential link between plant and fungal distributions in a dipterocarp rainforest: community and phylogenetic structure of tropical ectomycorrhizal fungi across a plant and soil ecotone. New Phytol 185: 529-542.

Posada, D., and Crandall, K.A. (1998) MODELTEST: testing the model of DNA substitution. Bioinformatics 14: 817-818.

Prosser, J.I., Bohannan, B.J.M., Curtis, T.P., Ellis, R.J., Firestone, M.K., Freckleton, R.P., et al. (2007) The role of ecological theory in microbial ecology. Nat Rev Microbio/ 5: 384-392.

Schloss, P.D., Westcott, S.L., Ryabin, T., Hall, J.R., Hartmann, M., Hollister, E.B., et al. (2009) Introducing mothur: open-source, platform-independent, communitysupported software for describing and comparing microbial communities. Appl Environ Microbiol 75: 7537-7541.

Stegen, J.C., Lin, X., Konopka, A.E., and Fredrickson, J.K. (2012) Stochastic and deterministic assembly processes in subsurface microbial communities. ISME J 6: 1653-1664.

Tamura, K., Peterson, D., Peterson, N., Stecher, G., Nei, M., and Kumar, S. (2011) MEGA5: molecular evolutionary genetics analysis using maximum likelihood, evolutionary distance, and maximum parsimony methods. Mol Biol Evol 28: 2731-2739.

Taylor, J.W., and Berbee, M.L. (2006) Dating divergences in the Fungal Tree of Life: review and new analyses. Mycologia 98: 838-849.

Tedersoo, L., Bahram, M., Toots, M., Diédhiou, A.G., Henkel, T.W., Kjøller, R., et al. (2012) Towards global patterns in the diversity and community structure of ectomycorrhizal fungi. Mol Ecol 21: 4160-4170.

Tilman, D. (1980) Resources: a graphical-mechanistic approach to competition and predation. Am Nat 116: 362393.

Timling, I., and Taylor, D.L. (2012) Peeking through a frosty window: molecular insights into the ecology of Arctic soil fungi. Fung Ecol 5: 419-429.

Treseder, K.K., Kivlin, S.N., and Hawkes, C.V. (2011) Evolutionary trade-offs among decomposers determine responses to nitrogen enrichment. Ecol Lett 14: 933-938.

Van der Heijden, M.G.A., Bardgett, R.D., and Van Straalen, N.M. (2008) The unseen majority: soil microbes as drivers of plant diversity and productivity in terrestrial ecosystems. Ecol Lett 11: 296-310.

Vellend, M. (2010) Conceptual synthesis of community ecology. Q Rev Biol 85: 183-206.

Webb, C.O., Ackerly, D.D., and Kembel, S.W. (2008) Phylocom: software for the analysis of phylogenetic community structure and trait evolution. Bioinformatics 24: 2098-2100.

Webb, C.O., Ackerly, D.D., McPeek, M.A., and Donoghue, M.J. (2002) Phylogenies and community ecology. Annu Rev Ecol Syst 33: 475-505.
Wilbur, H.M., and Alford, R.A. (1985) Priority effects in experimental pond communities: responses of hyla to bufo and rana. Ecology 66: 1106-1114.

Wu, L., Liu, X., Schadt, C.W., and Zhou, J. (2006) Microarraybased analysis of subnanogram quantities of microbial community DNAs by using whole-community genome amplification. Appl Environ Microbiol 72: 4931-4941.

Xie, J., He, Z., Liu, X., Liu, X., Van Nostrand, J.D., Deng, Y., et al. (2011) GeoChip-based analysis of the functional gene diversity and metabolic potential of microbial communities in acid mine drainage. Appl Environ Microbiol 77: 991-999.

Zak, D.R., Pregitzer, K.S., Burton, A.J., Edwards, I.P., and Kellner, H. (2011) Microbial responses to a changing environment: implications for the future functioning of terrestrial ecosystems. Fung Ecol 4: 386-395.

Zhang, Y., Zhang, X., Liu, X., Xiao, Y., Qu, L., Wu, L., and Zhou, J. (2007) Microarray-based analysis of changes in diversity of microbial genes involved in organic carbon decomposition following land use/cover changes. FEMS Microbiol Lett 266: 144-151.

\section{Supporting information}

Additional Supporting Information may be found in the online version of this article at the publisher's web-site:

Fig. S1. Overall metabolic potential, calculated as the corrected fluorescent signal intensity (SI) of all functional gene categories, significantly varied between sites (one-way ANOVA; $P=0.032$ ) and increased with site age. SI was corrected by the number of probes per gene as well as genes per gene category. Lowercase letters represent statistical differences. Error bars represent standard error.

Fig. S2. Geographic distance plotted against variation in soil climate (A), plant community composition (B) and biogeochemical characteristics (C). A positive trend was observed between pairwise geographic distance and biogeochemical distance. No significant relationship was found between geographic distance and soil climate $\left(r^{2}<0.001 ; \quad P=0.95\right)$ or plant community distance $\left(r^{2}=0.010 ; P=0.85\right)$.

Fig. S3. Maximum likelihood tree of Dikarya OTUs based on $28 \mathrm{~S}$ ribsomal gene. Phylogenetic tree was constructed in MEGA 5.0 using GTR subsititution method. Taxonomic assignments were based on reference sequences that can be found in Table S4. Bar length represents substitution rate.

Table S1. Relative abundance of plant composition based on basal areas of overstory tree species. $A B$, American beech; BC, black cherry; BF, balsam fir; BO = black oak; BW, American basswood; SM, sugar maple; RM, red maple; RO, red oak; IW, ironwood; YB, yellow birch; WA, white ash.

Table S2. Pairwise site comparisons of phylogenetic composition $\beta$-diversity based on two metrics: $\beta N T I$ and $D_{p}$. $\beta$-nearest taxon distance $(\beta \mathrm{NTI})$ calculates the minimum phylogenetic distance between an OTU in one community and all OTUs in a second community, and then determines the number of standard deviations from a null model community. $\beta N T I$ significantly greater than zero suggests phylogenetic $\beta$-diversity is greater than expected for one 
1548 L. C. Cline and D. R. Zak

pairwise comparison, and less than zero suggests lower than expected phylogenetic $\beta$-diversity. Rao's phylogenetic distance $\left(D_{p}\right)$ calculated the pairwise differences in branch length of OTUs in a community relative to another. A higher $D_{p}$ value for a pairwise comparison is indicative of higher phylogenetic distance between sites.
Table S3. Summary of gene categories used in functional gene microarray analysis from the GEOCHIP 4.0 based on depolymerization of components found in plant litter.

Table S4. Accession numbers and taxonomy of references sequences used for sequence alignment and phylogenetic tree construction. 